\title{
Menopausal osteoporosis: screening, prevention and treatment
}

\author{
Eu-Leong Yong ${ }^{1,2}$, FRCOG, PhD, Susan $\underline{\text { Logan }}^{1,2}$, FRCOG, FFSRH
}

\begin{abstract}
Screening for osteoporosis in women can be based on age and weight, using the Osteoporosis Screening Tool for Asians and assessment for other risk factors such as early menopause, Chinese ethnicity and other secondary factors. Based on the resulting risk profile, women can be triaged to dual-energy X-ray absorptiometry (DEXA) scanning for definite diagnosis of osteoporosis. Treatment should be considered in women with previous fragility fractures, DEXA-diagnosed osteoporosis and high risk of fracture. Exercise improves muscle function, can help prevent falls and has moderate effects on improvements in bone mass. Women should ensure adequate calcium intake and vitamin D. Menopausal hormone therapy (MHT) effectively prevents osteoporosis and fractures, and should be encouraged in those aged $<50$ years. For women aged $<60$ years, MHT or tibolone can be considered, especially if they have vasomotor or genitourinary symptoms. Risedronate or bisphosphonates may then be reserved for those aged over 60 years.
\end{abstract}

Keywords: menopause, osteoporosis, prevention, screening, treatment

\section{INTRODUCTION}

Osteoporosis is of clinical importance because it is the major risk factor for fractures. Osteoporotic fractures of the hip, spine, and forearms are associated with limitation of ambulation, physical deformity, chronic pain and disability, loss of independence, and decreased quality of life, both in Singapore ${ }^{(1)}$ and globally..$^{(2,3)}$ Osteoporotic hip fractures are especially devastating, contributing up to $5 \%$ of all-cause mortality for men and women combined, ${ }^{(4)}$ with $21 \%-30 \%$ dying within one year. ${ }^{(5)}$

\section{Hospital admissions for hip fractures projected to increase with ageing population}

Due to an ageing population, hip fracture admissions to Singapore hospitals have increased exponentially in the new century. Rates are two- to threefold higher for every additional five years of age. ${ }^{(6)}$ For Singapore women, absolute fracture numbers have increased by $3.3 \%(95 \%$ confidence interval $[\mathrm{Cl}] 3.0-3.6)$ annually, leading to an absolute average increase of 46.3 (95\% Cl 41-52) fractures/ year (Fig. 1a). ${ }^{(6)}$ However, a decreasing trend was observed when fractures rates were age-adjusted and expressed per 100,000 population (Fig. 1b), indicating the influence of ageing on the increase in absolute numbers. Following a hip fracture in Singapore, the risk of death is double (standardised mortality ratio 2.05) that of the age-matched general population in the first year, and this excess death risk was still present more than a decade later. ${ }^{(7)}$ The morbidity and mortality risks after hip fractures will place a huge burden on Singapore's healthcare system in the years to come. New preventive strategies need to be in place to arrest these trends.

\section{Women affected by osteoporosis and fractures due to rapid bone loss in perimenopause}

Due to increasing life expectancy, more than one-third of the average Singapore woman's life takes place after menopause. The amount of bone mass reached at the time of menopause is key to subsequent risk of fracture. Cessation of ovarian function after menopause is associated with a precipitous decline in circulating oestrogen levels. Women have smaller and thinner bones than men, and the fall in oestrogen levels causes rapid acceleration in bone loss that starts the year before menopause and continues for another three years before de-accelerating slightly, with a moderate rate of bone loss in the subsequent $4-8$ years. ${ }^{(8)}$ The average decrease in bone mineral density (BMD) during the menopausal transition is about $10 \%$, meaning that half the women are losing bone even faster, up to a $20 \%$ loss in the 5-7 years around menopause. ${ }^{(9)}$ Furthermore, about $25 \%$ of postmenopausal women can be further classified as fast bone losers, as measured by bone loss rates and bone resorption markers. In Singapore, approximately $8 \%$ of midlife women have osteoporosis ${ }^{(10)}$ and two-thirds of all hip fractures occur in women (Fig. 1). ${ }^{(6)}$

\section{Diagnosing osteoporosis with bone mineral density scanning}

While the diagnosis of osteoporosis by the presence of a fragility fracture is universally accepted, measurement of BMD with dualenergy X-ray absorptiometry (DEXA) can accurately diagnose osteoporosis before a fracture occurs. ${ }^{(11)}$ Osteoporosis occurs when BMD falls below 2.5 standard deviations (SD) as compared to the $\mathrm{BMD}$ of healthy young women at peak bone mass. According to the World Health Organization, every SD decrease in femoral neck BMD T-scores is associated a 2.6-fold increased risk of hip fracture. ${ }^{(12)}$ Similarly, every SD decrease in BMD T-scores for the spine and distal radius increases the risk of fractures 2.3- and 1.7-fold, respectively. DEXA scanning, however, is costly at SGD 50-126 per scan and requires specialised equipment and trained staff. The cost-effectiveness of DEXA screening for a whole population at risk has not been demonstrated. ${ }^{(11,13)}$ Consequently, most countries do not recommend DEXA for population-based

${ }^{1}$ Department of Obstetrics and Gynaecology, National University Hospital, ${ }^{2}$ Yong Loo Lin School of Medicine, National University of Singapore, Singapore Correspondence: Prof Eu-Leong Yong, Emeritus Consultant, Department of Obstetrics and Gynaecology, National University Hospital, NUHS Tower Block, 1 E Kent Ridge Road, Level 12, Singapore 119228. obgyel@nus.edu.sg 

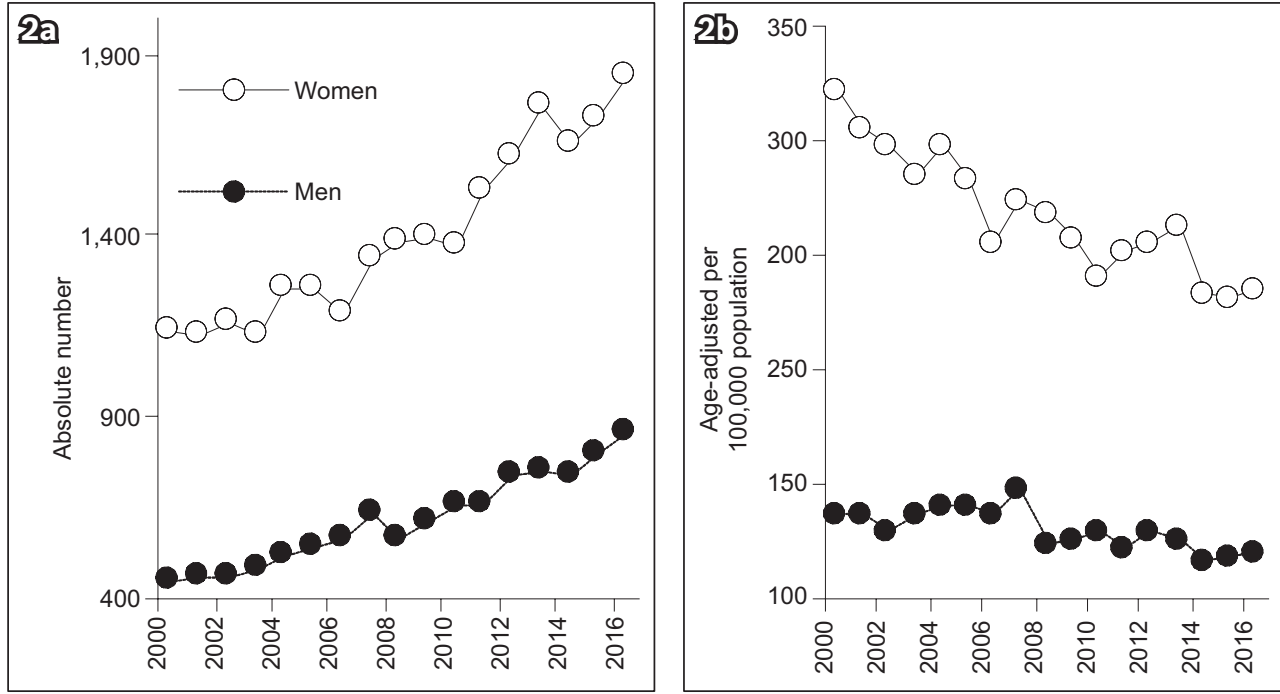

Fig. 1 Charts show hip fractures in Singapore from 2000 to 2017 in (a) absolute numbers and (b) age-adjusted rates per 100,000 population. (Reprinted with permission from Springer Nature Customer Service Centre GmbH: Springer Nature, Osteoporosis International, Hip fractures in Singapore: ethnic differences and temporal trends in the new millennium. Yong EL, 2019.)

screening, with many guidelines recommending DEXA scanning for women aged above 65 years. ${ }^{(3,11)}$

\section{Opportunistic screening of midlife women for osteoporosis}

For women aged below 65 years, most countries screen for osteoporosis based on an opportunistic case-finding strategy, ${ }^{(14)}$ wherein a formal clinical risk assessment tool is used to identify those at high risk of osteoporosis. ${ }^{(3,11,15)}$ Tools developed to identify women at increased risk of osteoporosis include SCORE (Simple Calculated Osteoporosis Risk Estimation), ${ }^{(16)}$ ORAI (Osteoporosis Risk Assessment Instrument), ${ }^{(17)}$ OSIRIS (Osteoporosis Index of Risk) ${ }^{(18)}$ and OST (Osteoporosis Self-assessment Tool). ${ }^{(19)}$ Singapore's guidelines ${ }^{(13,20)}$ recommend using the Osteoporosis Screening Tool for Asians (OSTA). This tool was developed specifically for Asian women and validated in an independent sample of 1,123 Japanese women. ${ }^{(21)}$ OSTA has an algorithm that gives individual risk scores for osteoporosis based on age and weight: score $=$ age (years) - weight (kg) (Fig. 2).

For women classified as high-risk for osteoporosis, DEXA should be considered, as the chance of finding osteoporosis is high. Those in the low-risk category can have their DEXA deferred. Risk for osteoporosis should be reassessed after five years if no clinical risk factors or significant weight loss develop since the preceding scan. Physicians should also consider a DEXA scan in women in the medium-risk category if they have other risk factors for osteoporosis, such as premature ovarian insufficiency (POI) before 40 years of age or early menopause (40-44 years of age) due to genetic, autoimmune, surgical or cancer treatment sequelae. Other risk factors include Chinese ethnicity, ${ }^{(10)}$ family history of osteoporosis or fractures, height loss $(>2 \mathrm{~cm}$ within three years), prolonged steroid use (> $5 \mathrm{mg} /$ day of prednisolone or its equivalent for $>3$ months in the past year), prolonged immobility, smoking, history of falls, excessive alcohol intake ( $>2$ units/day) and low calcium intake ( $<500 \mathrm{mg} /$ day). Secondary causes of osteoporosis, such as diabetes mellitus, rheumatoid arthritis or parathyroid disease, account for up to $45 \%$ of cases of osteoporosis in postmenopausal women. ${ }^{(22)}$ OSTA has a sensitivity and specificity of $91 \%$ and $45 \%$, respectively, and compares well to other more complicated tools such as SCORE, ${ }^{(16)}$ ORAI ${ }^{(17)}$ and OSIRIS. ${ }^{(18)}$

\section{Clinical risk assessment for osteoporosis using the Fracture Risk Assessment Tool}

Although osteoporosis can be diagnosed with clarity, it is fractures that are of public health concern. Furthermore, the majority of hip fractures occur in individuals without osteoporosis. Therefore, an osteoporosis risk tool based on T-scores may be inaccurate in measuring fracture risk, as clinical risk factors contribute significantly to fracture risk over and above that provided by BMD T-scores. For instance, age affects the significance of any given T-score to fracture risk in women by five- to sixfold. ${ }^{(12)}$ Another approach to clinical risk screening is to predict fracture risk using tools such as the Fracture Risk Assessment Tool (FRAX®). FRAX (https://www.sheffield.ac.uk/FRAX/tool.aspx) calculates the patient's ten-year probability of having a fracture using a complex algorithm that includes country, age, body mass index, ethnicity (Singapore-specific) and other risk factors. FRAX can be used with or without BMD measurements.

The question arises as to why a tool for fracture risk assessment has not been adopted in Singapore. Fractures occur for many reasons other than osteoporosis. These factors include propensity for falls due to impaired mental status, cognitive and motor deficits, poor vision and balance, disequilibrium due to medications, and reduced muscle strength due to frailty. Fracture risk prediction also requires intervention thresholds that depend on socioeconomic conditions specific to each population. These conditions include overall wealth, the fraction of gross domestic product spent on healthcare, drug affordability, life expectancy with and without fractures, the cost of fracture treatment and rehabilitation and quality of life after fractures. The risk threshold for interventions may therefore vary widely from one country to 


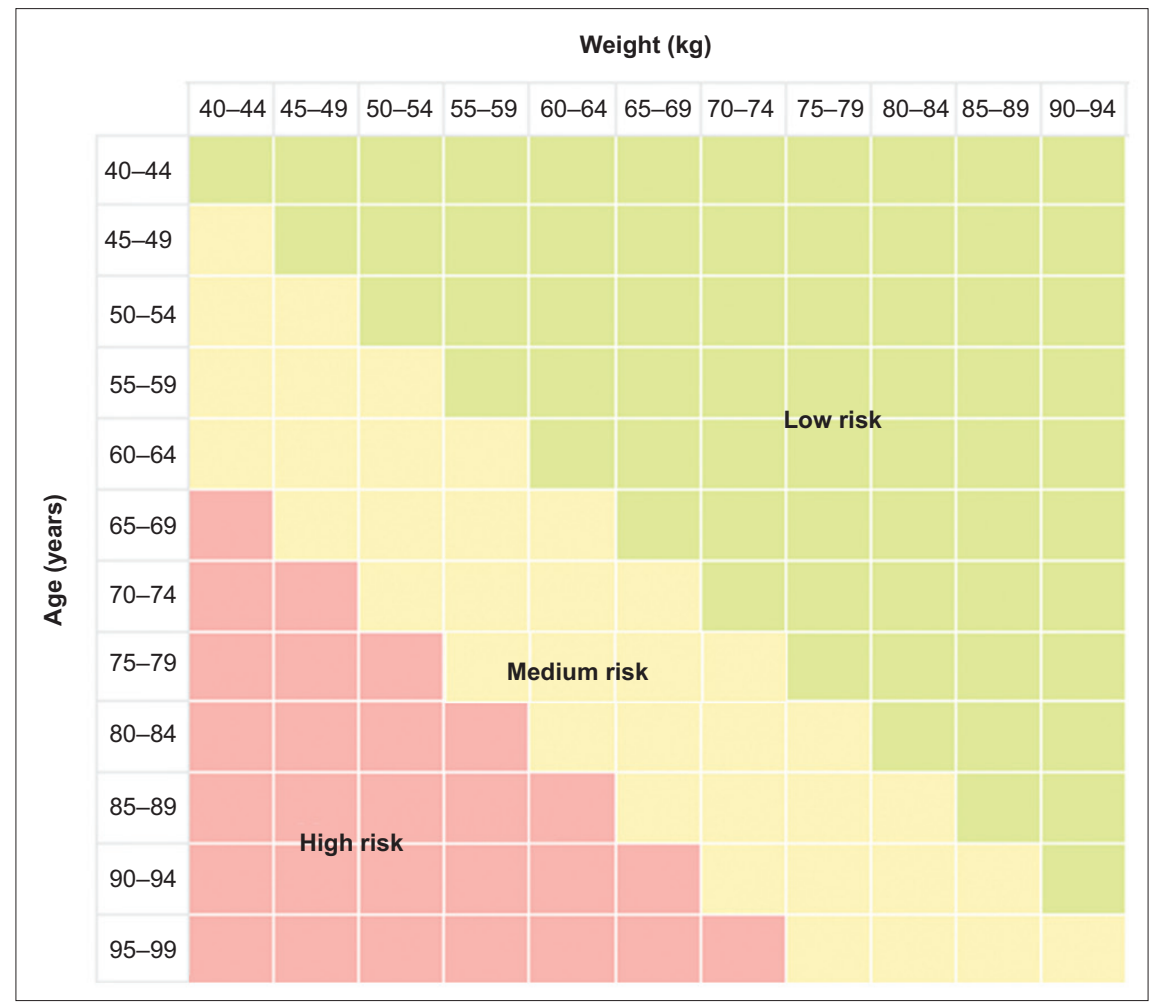

Fig. 2 Diagram shows osteoporosis risk stratification for Singapore women based on the Osteoporosis Screening Tool for Asians. ${ }^{(20)}$

another. Finally, all randomised controlled trials (RCTs) of drug treatments enrolled women based on the presence of osteoporosis as assessed with BMD. ${ }^{(12)}$ In practice, it is not feasible to reverse the majority of fracture risk factors, so the potential effect of their modification at the population level is substantially smaller than for osteoporosis, for which specific pharmacological interventions have proven effective. Application of United States (US) and Canadian osteoporosis screening strategies indicate that the FRAX and Garvan fracture risk calculators without BMD have specificity of $<50 \%$ and both failed to identify the majority of women who actually experienced major osteoporotic fractures during ten years of follow-up in the large Women's Health Initiative (WHI) study. ${ }^{(22)}$ Nevertheless, determining fracture risk through FRAX can assist in clinical decision-making regarding treatment. Singapore-specific thresholds for treatment have been calculated at $14.0 \%$ and $3.5 \%$ for major and hip osteoporotic fractures, respectively. ${ }^{(23)}$

\section{Is population-based osteoporosis screening cost-effective?}

No nation has reported on the cost-effectiveness of osteoporosis treatment and screening on a population basis. Even modelling for the incremental cost-effectiveness of osteoporosis screening at a population level is a complex exercise. ${ }^{(14,24)}$ Cost-effective screening depends on the prevalence of osteoporosis. A Japanese model-based study suggested that the cost-effectiveness of screening improves with increasing age. ${ }^{(25)}$ The SCOOP (screening for prevention of fractures in older women) RCT from the United Kingdom (UK) observed that with a 10\% fracture probability, hip fractures in elderly women were not significantly reduced in the intervention group compared to the control group, whereas with a $90 \%$ risk, the intervention group (vs. control group) experienced a significant $33 \%$ reduction in hip fractures. ${ }^{(26,27)}$ Similarly, the ROSE RCT from Denmark observed a significant reduction in fracture risk in older women, with adjusted hazard ratios ranging from 0.74 to 0.89 , limited to those at higher risk of fractures. ${ }^{(28)}$ Data on the cost-effectiveness of screening at various levels of osteoporosis risk is very limited, ${ }^{(14,24)}$ and this knowledge gap needs to be urgently addressed. In fact, the UK National Screening Committee in $2019^{(29)}$ stated that systematic population screening is not recommended because: (a) it is not known how accurate screening tests were for the women who were screened; (b) it is not known what effect treatment and changes in lifestyle would have on some types of fracture; (c) it is not known what effect treatment and changes in lifestyle will have on women who are identified through screening as being at risk of fracture; (d) research shows that screening all women does not reduce the number of fractures compared to current care; and (e) although there is some evidence that hip fractures may be reduced by screening, more research is needed to understand if this is correct.

\section{TREATMENT OF OSTEOPOROSIS}

Guidelines for drug interventions for osteoporosis are clear and have been shown to be cost-effective on a population basis. ${ }^{(30,31)}$ Treatment can be considered if the woman has a previous fragility fracture, ${ }^{(32)}$ a DEXA BMD T-score $\leq-2.5$, or T-scores between -1 and -2.5 with high fracture risk.

\section{Non-pharmacological options \\ Exercise}

Given that fractures result from falls, improving muscle tone and balance through exercise can reduce the risk of falls. Resistance exercises have significant low-to-moderate effects 
on BMD changes in postmenopausal women. ${ }^{(33)}$ Both high-load and low-load resistance training appear equally effective and have similar effects on femoral neck and lumbar spine BMD in ageing people. ${ }^{(34)}$ Balance exercises popular in Singapore such as qigong, ${ }^{(35)}$ tai chi and yoga can improve muscle tone and mental health, both of which are factors that affect one's risk of osteoporosis. High-intensity resistance and impact training has recently been shown to be efficacious and induced no adverse events under highly supervised conditions in otherwise healthy postmenopausal women with low to very low bone mass. ${ }^{(36)}$ However, many Singaporean women are sedentary, and it may be unpalatable for many women in hot tropical Singapore to exercise. Many women also complain of joint pains and knee problems that discourage exercise. New programmes and innovations for easily accessible exercise programmes are required, especially in this era of COVID-19 and social distancing.

\section{Calcium and vitamin D}

The value of calcium and vitamin D administration for prevention of osteoporotic fractures is unclear. In Singapore, vitamin D is derived mainly from cutaneous synthesis, with Chinese women being the most vitamin D replete and Indian women being at risk of vitamin $\mathrm{D}$ deficiency. ${ }^{(37)}$ In a large randomised trial by WHI investigators involving more than 36,000 postmenopausal women, calcium (1,000 mg of elemental calcium supplementation daily) plus vitamin D (400 IU daily) did not have a significant effect on fractures. ${ }^{(38)}$ On the other hand, large doses of vitamin D have been shown to increase the risk of fractures and are not recommended. ${ }^{(11)}$ In the WHI trial, women assigned to the calcium with vitamin D group had a $17 \%$ higher risk of kidney stones than women assigned to a placebo, most likely owing to a high intake of calcium at baseline (approximately 1,150 mg/day in each group). For optimal bone health, women should have a total calcium intake of 1,000-1,500 mg/day, preferably through their diets. Supplementation of vitamin D of $600-800$ IU/day is indicated in those with deficient cutaneous synthesis.

\section{Reducing risk of falls at home}

Besides exercise, assessment of the home for hazards, appropriate footwear, withdrawal of 'at risk' medications (when possible), and the use of a multidisciplinary programme to assess risk factors are prudent strategies for potentially reducing the risk of falls.

\section{Pharmacological therapies}

Since osteoporosis risk increases steeply with oestrogen withdrawal at menopause, it stands to reason that oestrogen replacement should first be considered for management of osteoporosis in midlife women. ${ }^{(39)}$ This was the case prior to the epochal WHI study in 2002, which reported that the health risks of menopausal hormone therapy (MHT) outweigh its benefits. ${ }^{(40)}$ In recent years, there has been a reconsideration of the data, leading to a more nuanced view of the complex pattern of risks and benefits of MHT. ${ }^{(41,42)}$ The concept of a 'window of opportunity' in the ten years after menopause and before 60 years of age has gained recognition. ${ }^{(43)}$ The use of MHT in healthy women before the age of 60 years did not increase all-cause mortality in the WHI study (Fig. 3). These findings led to the following age-based schema for consideration of MHT and other treatment modalities for pharmacological treatment of osteoporosis in midlife women.

\section{Before 50 years of age: oestrogen replacement encouraged}

Oestrogen is one of the very few drugs with both anabolic and anti-resorptive effects on bone cells.(2) Randomised, controlled trials and observational studies show that standard-dose MHT reduces hip fractures by $28 \%$ (relative risk [RR] 0.72 [0.53-0.98]), vertebrae fractures by $35 \%$ (RR 0.65 [0.46-0.92]) and other non-vertebral fractures by $27 \%$ (RR 0.73 [0.58-0.94]). ${ }^{(44)} \mathrm{MHT}$ utilises lower levels of hormones compared to oral contraceptive formulations that require supraphysiological doses to suppress ovulation, increasing its level of safety. The benefit-risk ratio is most favourable for women with oestrogen deficiency due to POI and early menopause. These women, especially those who experience surgical menopause, frequently suffer from distressing vasomotor symptoms, which MHT largely resolves. MHT also protects against genitourinary syndrome of menopause (GSM), which affects the lower genitourinary tract and is characterised by vulval itch, vaginal dryness, dyspareunia, urinary frequency, urgency, nocturia, urge incontinence and urinary tract infection. Therapy should continue until at least the age of menopause (49 years in Singapore). Observational studies suggest that benefits outweigh risks for effects on bone, heart, cognition, genitourinary symptoms, sexual function, mood and quality of life. ${ }^{(39)}$

Women without a uterus should receive oestrogen alone. Natural oestrogens administered non-orally in the form of transdermal patches or gels offer advantages such as no increased risk of stroke, venous thromboembolism (VTE) and gallstones through bypassing the first-pass hepatic effect. ${ }^{(45)}$ However, there are no head-to-head RCTs to validate this effect. Conjugated equine oestrogens (CEEs), as used by the $\mathrm{WHI}$, are isolated from the urine of pregnant mares and comprise estrone sulfate (weaker than estradiol) and mixtures of more than ten minor components of different active forms of oestrogens. Other common oestrogen preparations include estradiol hemihydrate and valerate. Ethinyl estradiol is a synthetic oestrogen. Ultra-low dose oestrogen $(0.014 \mathrm{mg} /$ day) has been shown to prevent reductions in BMD in postmenopausal women without causing uterine hyperplasia and has been approved by the US Food and Drug Administration (FDA). ${ }^{(46)}$

Progestogen is needed in women with intact uteri, as chronic unopposed oestrogen exposure increases the risk of endometrial hyperplasia and cancer. Commonly used progestins include medroxyprogesterone acetate (MPA), norethindrone acetate and natural progesterone. When adequate progestogen is combined with oestrogen sequentially (10-14 days per month), the risk of endometrial neoplasia is not higher than in untreated women. ${ }^{(47)}$ If the progestogen is taken continuously, the risk is lower. Regarding breast risk, the WHI saw a higher incidence of breast cancer for CEE and MPA compared with a placebo, but a reduced incidence with CEE alone. ${ }^{(48)} \mathrm{A}$ recent meta-analysis of worldwide epidemiological evidence concluded that taking five years of MHT at 50 years of age would increase breast 

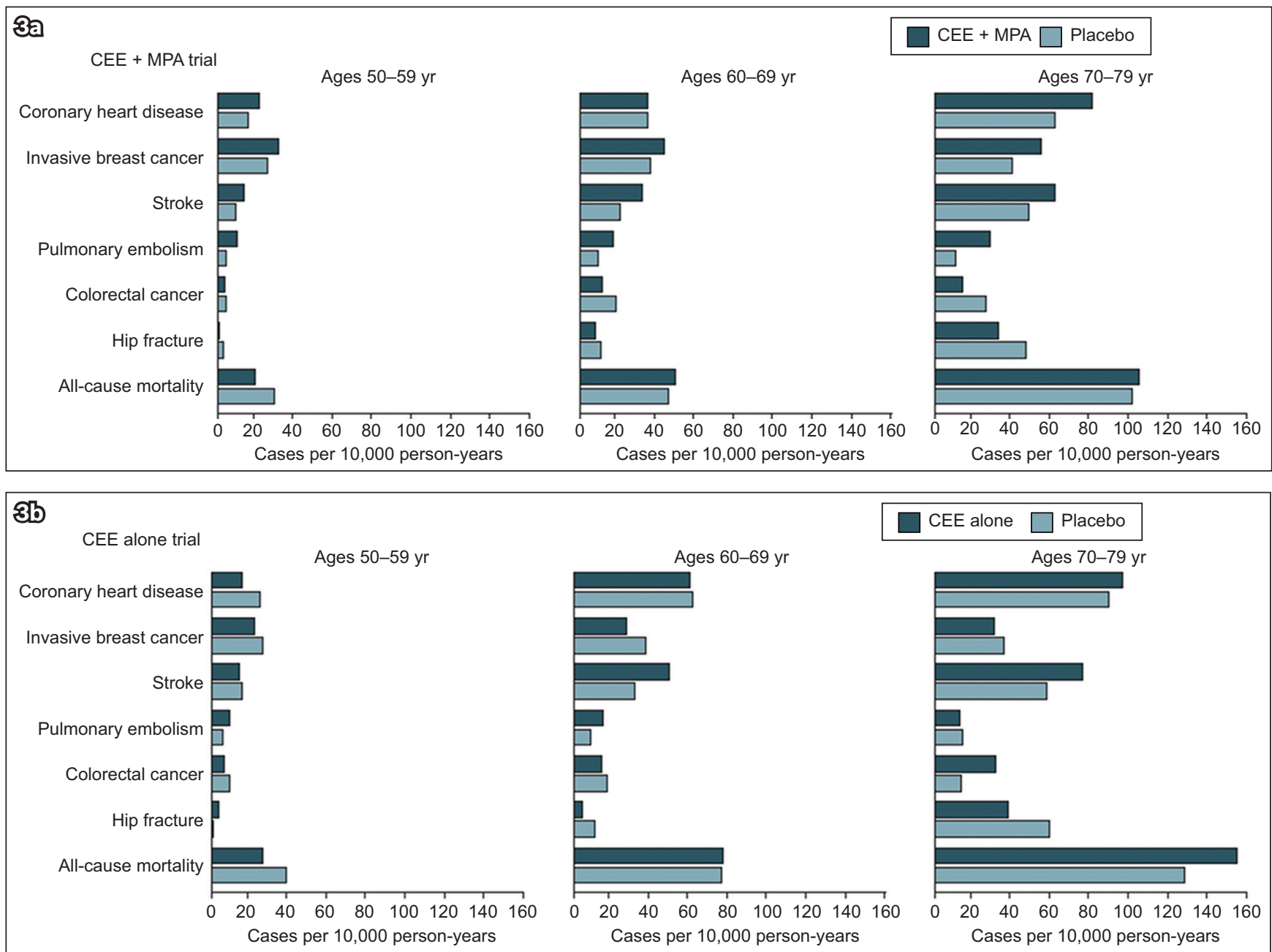

Fig. 3 Charts show the absolute risks of health outcomes by ten-year age groups in (a) the CEE-MPA trial and (b) the CEE alone trial of the Women's Health Initiative hormone therapy trials during the intervention phase. (Reproduced with permission from JAMA 2013; 310:1353-68. Copyright (c) 2013 American Medical Association. All rights reserved.) CEE: conjugated equine oestrogens; MPA: medroxyprogesterone acetate

cancer incidence at 50-69 years by: about one in every 50 users of oestrogen plus daily progestogen; one in every 70 users of oestrogen plus intermittent progestogen; and one in every 200 users of oestrogen-only preparations. There was no increased risk for oestrogen alone if only RCTs were analysed. ${ }^{(49)}$

\section{Routes of administration}

Systemic oestrogen can be prescribed as oral tablets, transdermal patches, sprays and gels. Transdermal administration avoids the first-pass hepatic effect on coagulation factors with no increased risk of stroke, VTE and gallstones. However, there are no head-to-head RCTs to validate this supposition. Progestogens are available as oral tablets and capsules or combined with oestrogen in patches, intrauterine systems, injectables, and vaginal gels or tablets.

\section{Safety considerations}

Across all ages, the $\mathrm{WHI}$ reported that MHT use was associated with an extra six strokes per 10,000 women (RR 1.24, 95\% Cl [1.10-1.41]), eight cases of VTE per 10,000 women (RR 1.92, $95 \% \mathrm{Cl}$ [1.36-2.69]), and four cases of pulmonary embolism per 10,000 women (RR 1.81, 95\% Cl [1.32-2.48]) compared with a placebo. ${ }^{(50)}$ Nonetheless, the same review did not find any increased risk of stroke in women aged younger than 60 years or within ten years of menopause. Newer observational data and reanalysis of older studies by age or time since menopause, including the $\mathrm{WHI}$, suggest that for healthy, recently menopausal women aged below 60 years, the benefits of MHT (oestrogen alone or with a progestogen) outweigh its risks, with fewer cardiovascular events in younger women compared to older women (Fig. 3).

Potential contraindications for MHT include unexplained vaginal bleeding, severe active liver disease, prior oestrogensensitive breast or endometrial cancer, coronary heart disease, stroke, dementia, personal history or inherited high risk of thromboembolic disease, and hypertriglyceridaemia. There is also a risk of reactivation of endometriosis, worsening of migraine headaches, or growth of leiomyomas. Women with complex medical conditions should be referred for specialist review. Common side effects include nausea, bloating, weight gain, fluid retention, mood swings, breakthrough bleeding (first 3-6 months), headaches and breast tenderness.

\section{0-59 years: consider menopausal hormone therapy}

MHT can be recommended to women in the 50-59 years age group, especially those with menopausal symptoms and GSM. 
The WHI trials identified no significant increased risk of heart disease in women randomised to receive oestrogen alone or oestrogen-progestogen therapy who were younger than 60 years or within ten years of menopause (Fig. 3). Although risk of stroke increased with oral $\mathrm{MHT}$, it is a rare event in this age group, with an absolute attributable risk of less than 0.5 additional cases per 1,000 women per year. ${ }^{(39)}$ Venous thrombotic events also increased with oral MHT use, but large observational studies do not identify increased risk with transdermal estradiol. ${ }^{(45)}$

Selective oestrogen receptor modulators (SERMs) activate distinct tissue receptors for oestrogen. Raloxifene, a SERM that is FDA-approved to treat osteoporosis, inhibits bone resorption, increases spine BMD and decreases the risk of vertebral fractures by $40 \%$. Nonvertebral or hip fractures are not reduced. ${ }^{(44)}$ Longterm use of raloxifene decreases breast-cancer risk among highrisk women but increases the risk of thromboembolic events.

Tibolone is a synthetic steroid with oestrogenic, progestogenic and androgenic properties. The effect on bone is similar to that of MHT and bisphosphonates with 30\% lower risk of hip fractures (RR 0.69 [0.32-1.51]). ${ }^{(51)}$ For menopausal symptoms, tibolone is more effective than a placebo but less effective than MHT. The side effects and contraindications are the same as those of oral MHT.

\section{Over 60 years: bisphosphonates preferred, use hormone therapy with caution}

Although hormone therapy can still be considered in those with troublesome vasomotor or GSM symptoms, it should be avoided in those at higher risk of breast cancer, ${ }^{(52)}$ cardiovascular disease or VTE. ${ }^{(53)}$ Although meta-analyses indicate that oestrogen or hormone therapy after 60 years did not affect risk of CHD (RR 1.07, 95\% Cl [0.96-1.20]) or all-cause mortality (RR 1.06, 95\% Cl [0.95-1.18]), the risk of stroke (RR 1.21, 95\% Cl [1.06-1.38]) and VTE (RR 1.96, 95\% Cl [1.37-2.80]) increased. ${ }^{(50)}$

Bisphosphonates may be the preferred intervention in women after 60 years. Bisphosphonates as a class represent the vast majority of prescriptions for osteoporosis treatment and are available in generic preparations. Bisphosphonates inhibit bone remodelling, and all oral bisphosphonates have been shown in randomised trials to reduce the risk of fractures. Although data from randomised trials and clinical experience indicates that they are generally safe, gastrointestinal irritation and muscle pain commonly occur. Two rare but more serious adverse effects have also been observed. These are atypical femoral fractures (i.e. fractures in the shaft of the femur that have a transverse orientation and non-comminuted morphologic features, show focal lateral cortical thickening, occur with minimal trauma, and may be bilateral) and osteonecrosis of the jaw, which is defined as exposed bone in the maxillofacial region that does not heal within eight weeks. Risks are higher if use is prolonged and assessment for drug holidays has been recommended after 1-2 years for risedronate, 3-5 years for alendronate and 3-6 years for zoledronic acid. ${ }^{(54)}$ If a new fracture is experienced, fracture risk has increased or BMD remains low (femoral neck T-score $\leq-2.5$ ), anti-osteoporotic treatment should be resumed.
Oral bisphosphonates are now used in weekly doses (alendronate and risedronate) or monthly doses (ibandronate and risedronate). Gastrointestinal irritation may be minimised by adherence to dosing instructions, switching to an effervescent form or using risedronate. However, adherence to oral bisphosphonates is low, with less than $40 \%$ of persons who are prescribed oral preparations taking them after one year. ${ }^{(41)}$ Intravenous bisphosphonates (ibandronate and zoledronic acid) are alternatives for poorly compliant patients. Use of bisphosphonates should be limited to persons with adequate renal function (creatinine clearance $>35 \mathrm{~mL}$ per minute) and normal serum vitamin D levels.

\section{Denosumab}

Denosumab was the first of a class of monoclonal antibodies that were approved for osteoporosis. Its action is distinct from that of bisphosphonates as it inhibits bone resorption by binding to the receptor activator of nuclear factor- $\kappa \beta$ ligand to reduce activation of osteoclasts and bone resorption. Unlike bisphosphonates, it can be used in women with compromised renal function. A large trial involving women with a BMD T-score of less than -2.5 but not less than -4.0 at the lumbar spine or total hip showed that treatment with denosumab $(60 \mathrm{mg}$ administered twice yearly by subcutaneous injection) resulted in a significantly lower risk of vertebral fractures (by 68\%), hip fractures (by 40\%), and nonvertebral fractures (by 20\%) compared to a placebo. ${ }^{(55)}$ As with bisphosphonates, rare cases of atypical femur fractures and osteonecrosis of the jaw have been observed. Recent concerns about rapid rebound bone loss following cessation of denosumab therapy, exceeding those on placebo, ${ }^{(54)}$ necessitate re-examination of its costs and benefits. ${ }^{(56)}$

\section{Teriparatide}

Teriparatide is one of the rare anabolic agents that works primarily by increasing bone formation rather than by decreasing resorption. The drug should be reserved for patients at very high risk for fractures, especially vertebral fractures, due to its cost and need for daily injections. In a 21-month trial involving women with low BMD and previous vertebral fractures, teriparatide (20 $\mu \mathrm{g} /$ day) was associated with a lower risk of vertebral fractures (by 65\%) and nonvertebral fractures (by 35\%) than the risk with a placebo, but not with a lower risk of hip fractures. ${ }^{(57)}$ After teriparatide is discontinued, its benefits are quickly lost and thus it should be followed by an antiresorptive agent. As osteosarcomas have been observed with long-term and high-dose administration, its use should be limited to two years.

\section{CONCLUSION}

A Singapore baby girl born in 2020 has a life expectancy of 83.6 years, ${ }^{(58)}$ which makes her one of the longest-living humans on the planet. ${ }^{(4)}$ More than one-third of these years will be spent in menopause, where the precipitous decline in oestrogens results in accelerated bone loss, risk of osteoporosis and fracture.

Screening for osteoporosis in women can be based on age and weight, using OSTA, and risk factor assessment. The major 
Box 1. Menopausal hormonal therapy (MHT) and osteoporosis by age:

\section{$<49$ years - MHT encouraged}

- Low risks of thromboembolism, breast cancer and cardiovascular disease

- No liver or gallbladder disease

50-59 years - consider MHT vs. bisphosphonates

- Persistent vasomotor symptoms with genitourinary syndrome and low risk factors favour MHT

- Bisphosphonates for those with high risk factors for MHT

$>60$ years - bisphosphonates preferred

- MHT with caution for those with acceptable risk for

thromboembolism, breast cancer and cardiovascular disease

\section{All ages}

- Exercise (resistance and balance)

- Adequate vitamin D/calcium

risk factors for osteoporosis are age, low body mass index, early menopause, Chinese ethnicity and other secondary factors. Based on the resulting risk profile, women can be triaged to undergo DEXA scanning for definite diagnosis of osteoporosis. Women with previous fragility fractures should be offered therapy. In those with DEXA-diagnosed osteoporosis or low bone mass and high risk of fracture, treatment should be strongly considered. Exercise improves muscle function, can help prevent falls and has moderate effects on improvement of bone mass. Women should ensure adequate calcium intake. Vitamin D insufficiency can occur in those with reduced skin production due to increased pigmentation.

MHT effectively prevents osteoporosis and fractures in menopausal women. We recommend using the schema shown in Box 1. Before the age of 50 years, women are best served with oestrogen replacement in the form of systemic MHT until the average age of menopause, when treatment may be reassessed. ${ }^{(59)}$ For women younger than 60 years of age, or who are within ten years of menopause onset, MHT or tibolone can be considered, especially if they have vasomotor or genitourinary symptoms. When alternate osteoporosis therapies are not appropriate or cause adverse events, extended use of MHT is an option for women who are at high risk of osteoporotic fracture. Risedronate can be considered in those with vertebral osteoporosis and increased breast cancer risk. Bisphosphonates may then be reserved for those over the age of 60 years and for whom MHT is contraindicated or unsuitable.

Overall drug adherence remains a problem, and health professionals involved in caring for menopausal women have an important role to correct misconceptions and ensure that osteoporosis is diagnosed and treated according to national guidelines, ${ }^{(20)}$ in order to blunt the coming epidemic of fragility fractures as Singapore matures.

\section{ACKNOWLEDGEMENT}

This study was partially funded by a Singapore National Medical Research Council grant (reference no. NMRC/CSA-SI/0010/2017) to Yong EL.

\section{REFERENCES}

1. Chandran M, McCloskey EV, Thu WPP, et al. FRAX® based intervention thresholds for management of osteoporosis in Singaporean women. Arch Osteoporos 2018; 13:130

2. Black DM, Rosen CJ. Clinical practice. Postmenopausal osteoporosis. N Engl J Med 2016; 374:254-62. Erratum in: N Engl J Med 2016; 374:1797.

3. Camacho PM, Petak SM, Binkley N, et al. American Association of Clinical Endocrinologists/American College of Endocrinology Clinical Practice Guidelines for the diagnosis and treatment of postmenopausal osteoporosis-2020 update. Endocr Pract 2020; 26:564-70.

4. GBD 2017 Disease and Injury Incidence and Prevalence Collaborators. Global, regional, and national incidence, prevalence, and years lived with disability for 354 diseases and injuries for 195 countries and territories, 1990-2017: a systematic analysis for the Global Burden of Disease Study 2017. Lancet 2018; 392:1789-858. Erratum in: Lancet 2019; 393:e44.

5. Viswanathan M, Reddy S, Berkman N, et al. Screening to prevent osteoporotic fractures: updated evidence report and systematic review for the US Preventive Services Task Force. JAMA 2018; 319:2532-51.

6. Yong EL, Ganesan G, Kramer MS, et al. Hip fractures in Singapore: ethnic differences and temporal trends in the new millennium. Osteoporos Int 2019; 30:879-86

7. Yong EL, Ganesan G, Kramer MS, et al. Risk factors and trends associated with mortality among adults with hip fracture in Singapore. JAMA Netw Open 2020; 3:e1919706.

8. Greendale GA, Sowers M, Han W, et al. Bone mineral density loss in relation to the final menstrual period in a multiethnic cohort: results from the Study of Women's Health Across the Nation (SWAN). J Bone Miner Res 2012; 27:111-8.

9. Tella SH, Gallagher JC. Prevention and treatment of postmenopausal osteoporosis. J Steroid Biochem Mol Biol 2014; 142:155-70.

10. Thu WPP, Logan SJS, Cauley JA, Kramer MS, Yong EL. Ethnic differences in bone mineral density among midlife women in a multi-ethnic Southeast Asian cohort. Arch Osteoporos 2019; 14:80.

11. US Preventive Services Task Force; Curry SI, Krist AH, Owens DK, et al. Screening for osteoporosis to prevent fractures: US Preventive Services Task Force recommendation statement. JAMA 2018; 319:2521-31.

12. Kanis JA; World Health Organization Scientific Group. Assessment of osteoporosis at the primary health-care level. Technical Report. Sheffield, UK: World Health Organization Collaborating Centre for Metabolic Bone Diseases, University of Sheffield, 2007.

13. Academy of Medicine, Singapore. Report of the Screening Test Review Committee. March 2019. Available at: https://www.ams.edu.sg/view-pdf. aspx?file=media\%5c4817_fi_59.pdf\&ofile=STRC+Report+March+2019.pdf. Accessed December 7, 2020.

14. Nayak S, Edwards DL, Saleh AA, Greenspan SL. Systematic review and metaanalysis of the performance of clinical risk assessment instruments for screening for osteoporosis or low bone density. Osteoporos Int 2015; 26:1543-54.

15. Osteoporosis: assessing the risk of fragility fracture. NICE Clinical Guidelines, No. 146. London: National Institute for Health and Care Excellence (UK), 2017.

16. Lydick E, Cook K, Turpin J, et al. Development and validation of a simple questionnaire to facilitate identification of women likely to have low bone density. Am J Manag Care 1998; 4:37-48.

17. Cadarette SM, Jaglal SB, Kreiger N, et al. Development and validation of the Osteoporosis Risk Assessment Instrument to facilitate selection of women for bone densitometry. CMAJ 2000; 162:1289-94.

18. Sedrine WB, Chevallier T, Zegels B, et al. Development and assessment of the Osteoporosis Index of Risk (OSIRIS) to facilitate selection of women for bone densitometry. Gynecol Endocrinol 2002; 16:245-50.

19. Richy F, Gourlay M, Ross PD, et al. Validation and comparative evaluation of the osteoporosis self-assessment tool (OST) in a Caucasian population from Belgium. QJM 2004; 97:39-46.

20. Agency of Care Effectiveness, Ministry of Health, Singapore. Osteoporosis identification and management in primary care. November 2018. Available at: https://www.ace-hta.gov.sg/healthcare-professionals/ace-clinical-guidances(acgs)/details/osteoporosis-identification-and-management-in-primary-care. Accessed December 7, 2020.

21. Koh LK, Sedrine WB, Torralba TP, et al; Osteoporosis Self-Assessment Tool for Asians (OSTA) Research Group. A simple tool to identify Asian women at increased risk of osteoporosis. Osteoporos Int 2001; 12:699-705.

22. Crandall CJ, Larson J, Cauley JA, et al. Do additional clinical risk factors improve the performance of Fracture Risk Assessment Tool (FRAX) among postmenopausal women? Findings from the Women's Health Initiative observational study and clinical trials. JBMR Plus 2019; 3:e10239.

23. Chandran M, Ganesan G, Tan KB, Reginster JY, Hiligsmann M. Cost-effectiveness of FRAX®-based intervention thresholds for management of osteoporosis in Singaporean women. Osteoporos Int 2021; 32:133-44.

24. Rubin KH, Friis-Holmberg T, Hermann AP, Abrahamsen B, Brixen K. Risk assessment tools to identify women with increased risk of osteoporotic fracture: complexity or simplicity? A systematic review. J Bone Miner Res $2013 ; 28: 1701-17$. 
25. Yoshimura M, Moriwaki K, Noto S, Takiguchi T. A model-based costeffectiveness analysis of osteoporosis screening and treatment strategy for postmenopausal Japanese women. Osteoporos Int 2017; 28:643-52.

26. McCloskey E, Johansson H, Harvey NC, et al; SCOOP Study Team. Management of patients with high baseline hip fracture risk by FRAX reduces hip fractures-a post hoc analysis of the SCOOP Study. J Bone Miner Res 2018; 33:1020-6.

27. Turner DA, Khioe RFS, Shepstone L, et al; SCOOP Study Team. The costeffectiveness of screening in the community to reduce osteoporotic fractures in older women in the UK: economic evaluation of the SCOOP Study. J Bone Miner Res 2018; 33:845-51.

28. Rubin KH, Rothmann MJ, Holmberg T, et al. Effectiveness of a two-step population-based osteoporosis screening program using FRAX: the randomized Risk-stratified Osteoporosis Strategy Evaluation (ROSE) study. Osteoporos Int 2018; 29:567-78.

29. National Screening Committee, UK. The UK NSC recommendation on osteoporosis screening women after the menopause. Available at: https:// legacyscreening.phe.org.uk/osteoporosis. Accessed December 7, 2020.

30. Adler RA, El-Hajj Fuleihan G, Bauer DC, et al. Managing osteoporosis in patients on long-term bisphosphonate treatment: report of a task force of the American Society for Bone and Mineral Research. J Bone Miner Res 2016; 31:16-35. Erratum in: J Bone Miner Res 2016; 31:1910.

31. Hadji P, Aapro MS, Body JJ, et al. Management of aromatase inhibitor-associated bone loss (AIBL) in postmenopausal women with hormone sensitive breas cancer: joint position statement of the IOF, CABS, ECTS, IEG, ESCEO IMS, and SIOG. J Bone Oncol 2017; 7:1-12.

32. Beaudoin C, Jean S, Moore L, et al. Number, location, and time since prior fracture as predictors of future fracture in the elderly from the general population. J Bone Miner Res 2018; 33:1956-66.

33. Shojaa M, von Stengel S, Kohl M, Schoene D, Kemmler W. Effects of dynamic resistance exercise on bone mineral density in postmenopausal women: a systematic review and meta-analysis with special emphasis on exercise parameters. Osteoporos Int 2020; 31:1427-44.

34. Souza D, Barbalho M, Ramirez-Campillo R, Martins W, Gentil P. High and low-load resistance training produce similar effects on bone mineral density of middle-aged and older people: a systematic review with meta-analysis of randomized clinical trials. Exp Gerontol 2020; 138:110973.

35. Zhang YP, Hu RX, Han M, et al. Evidence base of clinical studies on Qi Gong: a bibliometric analysis. Complement Ther Med 2020; 50:102392.

36. Watson SL, Weeks BK, Weis LJ, et al. High-intensity resistance and impact training improves bone mineral density and physical function in postmenopausal women with osteopenia and osteoporosis: the LIFTMOR randomized controlled trial. Bone Miner Res 2018; 33:211-20. Erratum in: J Bone Miner Res 2019; 34:572.

37. Cheong WF, Ji S, Cazenave-Gassiot A, et al. Predictors of circulating vitamin D levels in healthy mid-life Singaporean women. Arch Osteoporos 2021; 16:26

38. Jackson RD, LaCroix AZ, Gass M, et al; Women's Health Initiative Investigators. Calcium plus vitamin D supplementation and the risk of fractures. N Engl J Med 2006; 354:669-83. Erratum in: N Engl J Med 2006; 354:1102.

39. Shifren JL, Crandall CJ, Manson JE. Menopausal hormone therapy. JAMA 2019 321:2458-9

40. Nelson HD, Humphrey LL, Nygren P, Teutsch SM, Allan JD. Postmenopausal hormone replacement therapy: scientific review. JAMA 2002; 288:872-81.

41. The NAMS 2017 Hormone Therapy Position Statement Advisory Panel. The 2017 hormone therapy position statement of The North American Menopause Society. Menopause 2017; 24:728-53.
42. Pinkerton JV, Kaunitz AM, Manson JE. Concern about US Preventive Services Task Force recommendation on hormone therapy for the primary prevention of chronic conditions in postmenopausal women. Menopause 2018; 25:476.

43. Davey DA. Menopausal hormone therapy: a better and safer future. Climacteric 2018; 21:454-61.

44. Barrionuevo P, Kapoor E, Asi N, et al. Efficacy of pharmacological therapies for the prevention of fractures in postmenopausal women: a network meta-analysis. J Clin Endocrinol Metab 2019; 104:1623-30.

45. Vinogradova Y, Coupland C, Hippisley-Cox J. Use of hormone replacement therapy and risk of venous thromboembolism: nested case-control studies using the QResearch and CPRD databases. BMJ 2019; 364:k4810. Erratum in: BMJ 2019; 364:1162.

46. Khosla S, Hofbauer LC. Osteoporosis treatment: recent developments and ongoing challenges. Lancet Diabetes Endocrinol 2017; 5:898-907.

47. Brinton LA, Felix AS. Menopausal hormone therapy and risk of endometrial cancer. J Steroid Biochem Mol Biol 2014; 142:83-9.

48. Manson JE, Chlebowski RT, Stefanick ML, et al. Menopausal hormone therapy and health outcomes during the intervention and extended poststopping phases of the Women's Health Initiative randomized trials. JAMA 2013; 310:1353-68.

49. Collaborative Group on Hormonal Factors in Breast Cancer. Type and timing of menopausal hormone therapy and breast cancer risk: individual participant meta-analysis of the worldwide epidemiological evidence. Lancet 2019; 394:1159-68.

50. Boardman HM, Hartley L, Eisinga A, et al. Hormone therapy for preventing cardiovascular disease in post-menopausal women. Cochrane Database Syst Rev 2015; (3):CD002229.

51. Formoso G, Perrone E, Maltoni S, et al. Short-term and long-term effects of tibolone in postmenopausal women. Cochrane Database Syst Rev 2016; 10:CD008536.

52. Le Ray I, Dell'Aniello S, Bonnetain F, Azoulay L, Suissa S. Local estrogen therapy and risk of breast cancer recurrence among hormone-treated patients: a nested case-control study. Breast Cancer Res Treat 2012; 135:603-9.

53. Rivera CM, Grossardt BR, Rhodes DJ, et al. Increased cardiovascular mortality after early bilateral oophorectomy. Menopause 2009; 16:15-23.

54. Anagnostis P, Paschou SA, Mintziori G, et al. Drug holidays from bisphosphonates and denosumab in postmenopausal osteoporosis: EMAS position statement. Maturitas 2017; 101:23-30.

55. Cummings SR, San Martin J, McClung MR, et al; FREEDOM Trial. Denosumab for prevention of fractures in postmenopausal women with osteoporosis. N Engl J Med 2009; 361:756-65. Erratum in: N Engl J Med 2009; 361:1914.

56. Tsourdi E, Langdahl B, Cohen-Solal M, et al. Discontinuation of denosumab therapy for osteoporosis: a systematic review and position statement by ECTS. Bone 2017; 105:11-7.

57. Neer RM, Arnaud CD, Zanchetta JR, et al. Effect of parathyroid hormone (1-34) on fractures and bone mineral density in postmenopausal women with osteoporosis. N Engl J Med 2001; 344:1434-41.

58. Department of Statistics, Singapore. Death and life expectancy 2020. Available at: https://www.singstat.gov.sg/find-data/search-by-theme/population/deathand-life-expectancy/latest-data. Accessed December 7, 2020.

59. Lobo RA, Pickar JH, Stevenson JC, Mack WJ, Hodis HN. Back to the future: hormone replacement therapy as part of a prevention strategy for women at the onset of menopause. Atherosclerosis 2016; 254:282-90.

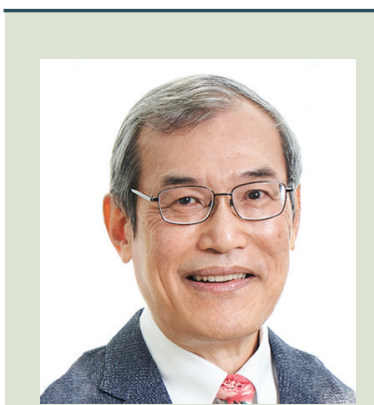

\section{About the First Author}

Prof Yong Eu Leong is Professor and Emeritus Consultant, Department of Obstetrics and Gynaecology, National University of Singapore and National University Hospital, Singapore. His subspecialty interests are reproductive endocrinology, polycystic ovarian syndrome, menopausal osteoporosis and ageing. He is one of the first clinician scientists in Singapore, with publications in high impact journals such as Lancet, Nature, Science, PNAS, EMBO and JAMA Open. He was awarded a Ministerial Citation by the National Science and Technology Board in 1999 and the National Outstanding Clinician Scientist Award in 2020. 